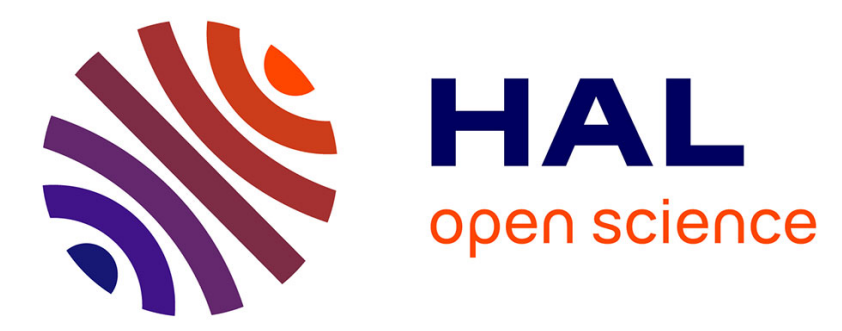

\title{
About the origin of the effect of super-deep penetration of powder particles into metallic target
}

\author{
S. Andilevko, O. Roman, S. Usherenko, V. Shilkin
}

\section{To cite this version:}

S. Andilevko, O. Roman, S. Usherenko, V. Shilkin. About the origin of the effect of super-deep penetration of powder particles into metallic target. Journal de Physique IV Proceedings, 1994, 04 (C8), pp.C8-795-C8-801. 10.1051/jp4:19948123 . jpa-00253366

\section{HAL Id: jpa-00253366 https://hal.science/jpa-00253366}

Submitted on 1 Jan 1994

HAL is a multi-disciplinary open access archive for the deposit and dissemination of scientific research documents, whether they are published or not. The documents may come from teaching and research institutions in France or abroad, or from public or private research centers.
L'archive ouverte pluridisciplinaire HAL, est destinée au dépôt et à la diffusion de documents scientifiques de niveau recherche, publiés ou non, émanant des établissements d'enseignement et de recherche français ou étrangers, des laboratoires publics ou privés. 


\title{
About the origin of the effect of super-deep penetration of powder particles into metallic target
}

\author{
S.K. Andilevko, O.V. Roman, S.M. Usherenko and V.A. Shilkin
}

Belorussian Research and Production, Powder Metallurgy Association, 220600 Minsk, Platonov str. 4I, Belarus

\begin{abstract}
The process known as Super-Deep Penetration (SDP) is discussed and a number of significant features associated with SDP and observed experimentally are described. A model for SDP, based on experimental data, is developed and relates to a channel-closing process behind the penetrating particles. The model has been validated numerically and predicts, with adequate accuracy, the effect of different loading conditions on the experimentally observed behaviour.
\end{abstract}

\section{INTRODUCTION}

Investigations of the interaction between a flux of powder particles and a metallic target have established that, under certain conditions, a finite proportion of these particles, about $0.1 \%$, penetrate the target to a depth, $h$, which equals or exceeds $10^{3} d_{p}$, where $d_{p}$ is the initial diameter of the particle. The effect may be observed for particles of diameter $d_{p} \leq 100 \mu \mathrm{m}$ travelling and impacting at a velocity, $U_{F}$, between 1 and $3 \mathrm{~km} / \mathrm{s}$ for targets of $\boldsymbol{F e}, \boldsymbol{C u}, \boldsymbol{T}, \boldsymbol{A l}, \boldsymbol{P b}$, etc, when, $\boldsymbol{\rho}_{F}$ the flux density, is equal to or exceeds $10^{3} \mathrm{~kg} \mathrm{~m}^{-3}$. The specific energy $\left(\mathrm{E}_{\mathrm{p}}=U^{2} / 2\right)$ for such particles does not exceed $5 \times 10^{5} \mathrm{~J} \mathrm{~kg}^{-1}$. This energy alone is insufficient for penetration to a depth greater than $50 d_{p}$. Penetration to depths of the order of $10^{3} d_{p}$, therefore, implies the existence of a new physical effect, known as Super-Deep Penetration (SDP).

Experimental investigations of SDP have revealed a number of significant features and dependencies of this effect:

- SDP occurs only when the flux density of the powder particles exceeds $0.25 \times 10^{3} \mathrm{~kg} \mathrm{~m}^{-3}$. It is not found for single particles or for low powder flux densities.

- When particles penetrate the target, zones of intensive plastic deformation are observed close to the path of penetration. The size of such zones, $d_{z}$, is found to depend on the particle size $\left(d_{z} \propto d_{p}\right)$. That part of these zones located closest to the path of penetration is found to be 
amorphous, ie, the metallic material has lost its crystalline structure.

- The motion of the particles penetrating the target under SDP depends on the mechanical and thermal properties of the target material but not on the configuration of its grain boundaries.

- The channels which are formed in the target by the passage of particles during their penetration close again after the particles have passed and can only be observed by sectioning and using a special etching and polishing technique.

- SDP is observed only if the particle size is smaller than a critical diameter $d_{c}$, where $d_{c} \sim 100$ $\mu \mathrm{m}$.

There have been many attempts to explain SDP. The most traditional explanation relates it to the propagation in the target of special cracks which must have particular properties; such cracks have not been observed up to the present time [1] and our own experimental investigations provides no evidence for the existence of such cracks, or even of ordinary cracks without particular properties, in a metallic target after SDP. On the contrary, SDP is observed only under the condition of a plastic interaction between the target and the particles.

The aim of this article, therefore, is the creation of an SDP model based on experimental data.

\section{A MODEL FOR SDP}

\subsection{Initial considerations}

When the target is loaded by the powder flux a pressure field is set up in the target, which consists of a long sequence of loading and partial unloading impulses. The intensity of each impulse is between 1 and $10 \mathrm{GPa}$, and the duration, $\tau$, is between $10^{-7}$ and $10^{-8} \mathrm{~s}$. There are between $10^{3}$ and $10^{5}$ such impulses during the interaction between the flux and the target. The specific energy transferred by each impulse to the target is about $10^{4} \mathrm{~J} \mathrm{~kg}^{-1}$. Even if the heat loss in this system is higher than usual the energy transferred by the total sequence of impulses would be enough to increase the specific energy of the target by $10^{6}$ to $10^{7} \mathrm{~J} \mathrm{~kg}^{-1}$, a value comparable to, or even higher than, the specific melting energy of the target:

$$
E_{m} \approx c_{p} T_{m}+L_{m} \propto 10^{6} \mathrm{~J} \mathrm{~kg}^{-1}
$$

where $c_{p}, T_{m}$ and $L_{m}$ are the specific heat, the melting temperature and the specific internal heat of melting. Hence the target material in the affected region, the size of which may be compared to the size of the powder flux, i.e., much greater than the size of a single particle, heats up to $T \approx T_{m}$. The state of the target material in such conditions may be characterized as unstable. In this state even a small increase in energy may lead to a total destruction of the metallic crystalline structure. The energy density in the zone of interaction between each particle and the target is much more than the specific energy of each impulse, $E_{p} \propto U^{2} / 2 \approx 10^{6} \mathrm{~J} \mathrm{~kg}^{-1}$. The time of interaction, $\tau_{i}$, is given by $\tau_{i} \propto$ $d_{p} / U$ and is comparable to the characteristic time of melting:

$$
\tau_{m} \approx(2 / 3) \lambda c_{p} \rho_{t}\left(T_{m}-T_{i}\right)^{2} / q^{2}
$$

where $q\left(=S t H_{0} U\right)$ is the heat flux per unit of contact surface, $S t$ is Stanton's number, $\boldsymbol{H}_{0}$ is the enthalpy of interaction, and $\boldsymbol{\lambda}$ is the heat conduction coefficient of the target. When:

$$
\tau_{i} \leq \tau_{R}=l^{2} / a, \quad a=\lambda /\left(\rho_{t} c_{p}\right)
$$

where $\tau_{R}$ is the relaxation time, $l$ is the width of the slip plane and $\rho_{t}$ is the density of the target, the 
main part of the interaction energy localizes in the zone of interaction. In this zone, the material will be softened or melted. The viscosity $\mu_{z}$ and hardness $\boldsymbol{H}_{z}$ of the material in this zone will be comparable to the viscosity $\mu_{m}$ and hardness $\boldsymbol{H}_{m}$ at melting. For steel these have the values $\mu_{m} \approx$ $2 \times 10^{-5} \mathrm{~m}^{2} \mathrm{~s}^{-1}$ and $\boldsymbol{H}_{m} \approx 0$.

The equation of motion for the particle in this case is given by:

$$
M_{p}(d U / d t)=-p_{F} S_{m i d F}+p_{R} S_{m i d R^{\prime}}
$$

where $M_{p}$ is the mass of the particle, $d U / d t$ is its acceleration, $p_{F}$ and $p_{R}$ are the pressures on the front and rear side of the particle and $S_{\text {midF }}$ and $S_{\text {midR }}$, are the effective areas of the front and rear cross sections of the particle. Provided that $R e$, the Reynolds number, approximately equals $10^{3}$ and $U<$ $c$, where $c$ is the sound speed of the target, then

$$
p_{F}=p+p_{t} U^{2} / 2
$$

where $p$ is the pressure in the target.

Even in this case, however, when we consider practically the penetration of a single particle into a melting metallic sample for which $p=0$ and $p_{R}=0$, the penetration depth will not be more than $20 d$. For penetration to a depth of between $h \approx 10^{3}$ and $10^{4} d_{p}$ each particle must have at least about 100 times more energy. But if the particle did have 100 times more energy before the interaction (or if this energy could be transferred in some way to the particle in a very short time during penetration), the interaction between target and particle would lead to a heat explosion which would completely destroy the particle. Therefore, some additional source of energy and a method of transferring it to the particle throughout the whole penetration process is required. The source of energy in this case may be only the energy of the powder flux which is being transferred during the interaction to the target and is accumulated in the structure of the target as the potential energy of the pressure field.

\subsection{The channel closing process}

Here it is proposed that the transmission of a part of this energy to a particle during its penetration into the target may be the result of the channel closing process, the occurrence of which, it should be noted, is an experimentally confirmed fact. Let us, therefore, consider the process of channel closing in detail.

If the pressure in the cavity immediately behind the particles $p_{c}$ is $<p$, where $p$ is the pressure in the target ahead of the particles, then the walls of the cavity will experience an effective pressure difference $\Delta p$ given by $\Delta p=p-p_{c}$ (see fig. 1). Under this pressure difference the cavity walls start to move radially inwards and converge at the point $\mathbf{O}$ in fig. 1 , where the angle of convergence is

$$
\alpha=\arccos \left[U /\left(U^{2}+W^{2}\right)^{1 / 2}\right]
$$

and $W(=W(\Delta p))$ is the radial velocity of the cavity walls. This velocity may be calculated using Bernouilli's law if the time of convergence $\tau_{c}<\tau_{t}$ where $\tau_{c} \approx d / W$ and $\tau_{t}$ is the total time of particle motion. Bernouilli's law in the present case may be written

$$
p_{c}+\rho_{t} \mu^{2} / 2=p+\rho_{t} U^{2} / 2
$$

so that

and

$$
u=U\left[1+2 \Delta p /\left(\rho_{t} U^{2}\right)\right]^{1 / 2}
$$


When the convergence angle $\alpha$ exceeds a given critical value $\alpha_{c}$, where for steel and aluminium $\boldsymbol{\alpha}_{c} \approx$ $20-30^{\circ} \quad[2]$, the flow originates at the point of convergence $\boldsymbol{O}$ to give an overtaking stream, OS, which propagates in the direction of particle motion. The conditions of formation and motion of such an overtaking stream are similar to those of a cumulative stream [3]. Its velocity in the coordinate system moving with the point $O$ is $V_{c s}=u$. The velocity of the point $O$ in the laboratory (unmoving) system is :

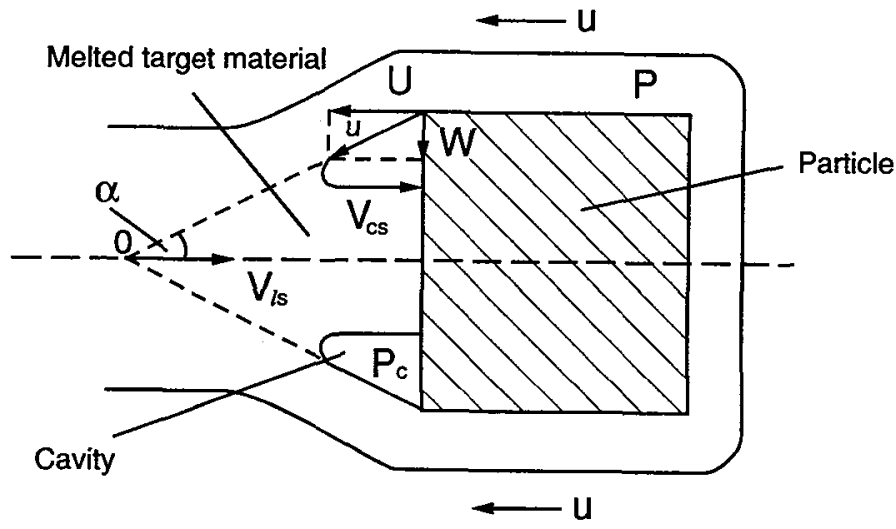

$$
\mathrm{V}_{\mathrm{ls}}=u / \cos \alpha=U\left(1+2 \Delta p / \rho_{t} U^{2}\right)
$$

so that the total velocity of the overtaking stream in the laboratory coordinate system is:

$$
V_{t}=V_{C S}+V_{l s}=U(1+\gamma)^{1 / 2}\left[1+(1+\gamma)^{1 / 2}\right]
$$

where $\gamma=2 \Delta p / \rho_{t} U^{2}$. Since $V_{t}>U$, the overtaking stream rapidly overtakes the particle in a time of the order $d / u \sim 10^{-9} \mathrm{~s}$ and transfers a part of its energy to the particle. The force acting on the trailing side of the particle is then given by

$$
p_{R} S_{m i d R^{\prime}}=S_{S} \rho_{t}\left(V_{t}-U\right)^{2}+p S_{S}+p_{c}\left(S_{m i d F}-S_{S}\right)
$$

where $S_{S}$ is the area of the point of contact between the particle and the overtaking stream. The velocity of the convergence point $\mathbf{O}$ is $V_{\boldsymbol{l}}>\boldsymbol{u}>\boldsymbol{U}$. Consequently, after a certain period of time, $\tau_{o S}$, of the order of $10^{-8}$ to $10^{-9} \mathrm{~s}$, the convergence point also merges with the trailing side of the particle and the cavity collapses completely. At that instant,

$$
S_{S} \propto S_{\text {midF }}, p_{c} \approx p, V_{t} \approx 2 U \text { and } p_{R} S_{m i d R^{\prime}} \approx S_{m i d F}\left(p+\rho_{\mathrm{t}} \mathrm{U}^{2} / 2\right) \equiv p_{F} S_{m i d F}
$$

The substitution of $p_{R} S_{\text {midR }}$ into equation (3) yields $M_{p}(d U / d t)=0$. Since $M_{p} \neq 0$, then $U=U_{*}=$ const. This means that from the instant the cavity closes completely and so long as the pressure $p$ exists in the target, the particle will move uniformly. The particle velocity is equal to that reached at the start of uniform motion and can be determined by integrating equation (3) under the additional conditions $p_{R}=0$ and $U\left(t=\tau_{x}\right)=U_{*}$. Then

$$
\begin{gathered}
U_{*}=\left(2 p / \rho_{t}\right)^{1 / 2} \tan \left\{\arctan [(1+1 / \gamma) \Gamma-1]^{1 / 2}-\rho_{t} d_{p} / 4 \rho_{p} l_{p}\right\} \\
\Gamma=\exp \left(-\rho_{t} / \rho_{p}\right)
\end{gathered}
$$

where $\rho_{p}$ and $l_{p}$ are the subsequent density and the length of the particle. Assuming the pressure field to be constant, the depth of penetration is about 


$$
h \approx U_{*}\left(\tau_{F l}-t_{i n}\right), \quad 0 \leq t_{i n} \leq \tau_{t}
$$

where $t_{i n}$ is the time from the start of flux interaction with the target to the instant of a complete collapse after the passage of the particle and $\tau_{F l}$ is the total time of the flux interaction with the target. It was possible, by calculation, to determine that at $p=3 \mathrm{GPa}, \rho_{t}=7830 \mathrm{~kg} \mathrm{~m}^{-3}, d=10 \mu \mathrm{m}$, $U=1500 \mathrm{~m} \mathrm{~s}^{-1}$ and $\rho_{p}=8900 \mathrm{~kg} \mathrm{~m}^{-3}$, the velocity $U_{*} \approx 974 \mathrm{~m} \mathrm{~s}^{-1}, \tau_{F l} \approx 3.5 \times 10^{-4} \mathrm{~s}$ and $t_{\text {in }}=0$. In this case the ultimate penetration depth of the particle $h \approx 0.033 \mathrm{~m}$ and $h / d \approx 3300$ in a total accordance with the experimental results.

\subsection{Generalized model for non-constant pressure}

The proposed model was developed for the simplest case where $p=$ const. Usually, however, $p=$ $p(r, t)$, where $r$ is the radial coordinate from the powder flux axis. In this more general case, the particle motion may be considered to be composed of a large number of regions of steady (uniform) and unsteady motion. The full depth of penetration can be determined by their successive summation:

$$
h\left(t_{i n}, r\right)=\int_{t_{i n}}^{\tau_{F}} U_{*}(t, r) d t
$$

If the dependence of pressure upon time is known, the depth of penetration of the particles may be calculated using this equation. The value of $t_{i n}$ determines the position of a particle in the flux. The dependence of penetration depth on $t_{i n}$ for the loading of an iron target by a $W$ powder flux with particle diameters $7 \mu \mathrm{m}$ $\leq d_{p} \leq 10 \mu \mathrm{m}$ is shown in fig. 2 . The maximum depth of penetration in this case is given by (13) for $t_{i n}=0$ and is approximately equal to $0.12 \mathrm{~m}$ for $h / d$ * 12000 to 16000 .

It was suggested in section 2.1 that the characteristic time of the particle interaction with the target $\tau_{t}$ should not exceed the time of thermal relaxation $\tau_{R}$, since otherwise it would be impossible to localize a sufficient amount of energy for softening the material in the interaction zone. Now, however, the total characteristic time of interaction, $\tau_{t}$, includes not only $\tau_{i}=d_{p} / U_{*}$, but also $\tau_{c}=d_{c} / W$, i.e.

$\left(h / d_{p}\right) 10^{-4}$

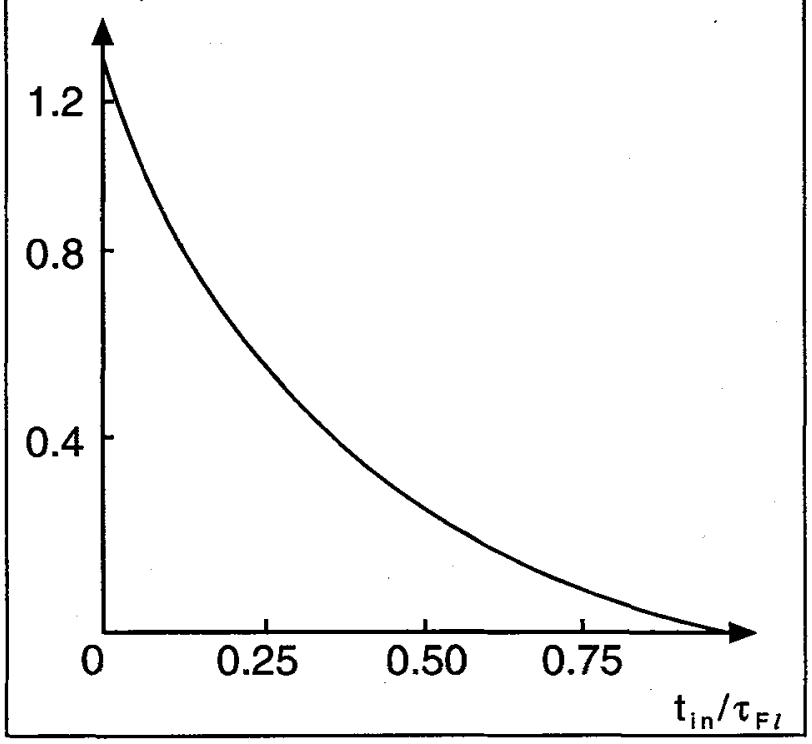

Fig. 2 The dependence of the (h/d) ratio on $t_{\text {in }}$ for tungsten powder particles penetrating an iron target.

the time of the cavity collapse. Thus, the necessary condition for SDP can be put inthe form of the inequality:

$$
\tau_{t}=\tau_{c}+\tau_{i} \leq \tau_{R}
$$

which after substitution from (2), gives

$$
d \leq d_{c} \text { and } d_{c} \approx\left(l^{2} / a\right)\left(2 p / \rho_{t}\right)^{1 / 2}(1+\gamma)^{-1}
$$


where $d_{c}$ is the critical size of the penetrating particles. Assuming that for a single particle, $p=0$, then $d_{c}=0$. Here the super-deep penetration of a single discrete particle into a metallic target is impossible. When $\boldsymbol{p} \Rightarrow \infty, \boldsymbol{d}_{c}$ approaches its limiting value

$$
d_{c} \approx\left(l^{2} / a\right) U
$$

From condition (15) it follows that the superposition of the additional pulsed (unsteady) pressure, synchronized with the flux of powder particles, should lead to an increase in $\boldsymbol{d}_{c}$ and, thus, to the enlargement of the range of particles for which the SDP effect can be observed. This, in turn, should cause an increase in the number of particles entering the metal. This was proved to be the case. A special experimental set-up was used in which the target was subjected to an additional loading by an explosive charge around its edges synchronised with the powder flux impacting its top surface at 1 to $3 \mathrm{~m} / \mathrm{s}$. When the pressure was raised by between 20 and 30\%, a 1.5 to 2 fold increase in the concentration of the material introduced was observed.

Another condition for penetration is $\alpha \geq \alpha_{c}$ or $\cos \alpha \leq \cos \alpha_{c}$. This suggests that the pressure generated in a target by a powder flux should obey the condition

$$
p \geq p_{c r}, \quad \text { where } p_{c r}=\rho_{t} U^{2} \tan \alpha_{c}
$$

Calculations show that for steel $d_{c} \approx 70$ to $90 \mu \mathrm{m}$ and $p_{c r} \approx 0.2$ to $0.8 \mathrm{GPa}$. While conditions (15) and (17) are the most essential for SDP, there are some other conditions which limit the parameters for SDP. Thus, for example, the velocity of uniform motion has to be limited and in particular, should be non zero, from which (11) gives:

$$
\left.0<d_{p} / l_{p} \leq\left(4 \rho_{p} / \rho_{t}\right) \arctan [(1+1 / \gamma) \Gamma-1)\right]^{1 / 2}
$$

\section{NUMERICAL VALIDATION OF MODEL}

The model developed above has been checked numerically using the method of "Large Particles" [4] and the FLIC (Fluid-in-Cell) method [5]. There is no room for a full discussion of the results here, but the main points developed in the model were confirmed. Penetration of the particles under pressures between 2.5 and 100 GPA was accomplished by the process of cavity collapse and the formation of an overtaking stream as proposed in the present model. The depth of penetration was about $1300 d_{p}$ and was limited only by the duration of the numerical calculation.

\section{CONCLUSIONS}

In summarizing the results, it should be pointed out that the assumption about the summative nature of SDP, i.e. when a larger portion of the flux, retarding on the target surfaces, transfers its energy to the target material where it is accumulated in the form of the pressure-field potential energy and, under conditions such as given in (15) and (17), can be subsequently transferred to certain particles to allow their deeper penetration, makes it possible not only to explain the SDP process, but also to predict with adequate accuracy the effect of the variation in the loading conditions on the experimentally observed behaviour. 


\section{REFERENCES}

1. S.S. Grigorian. About the Nature of Super-Deep Penetration of Solid Microparticles into Solid Materials. Docl. AN SSSR 292 (6), pp. 1319-1323 (1987).

2. S.A. Kinelovsky and Yu.A. Trishin. Physical Aspects of Cumulation, Fiz. Goren. Vzryva 19 (50), pp. 26-40 (1980).

3. M.A. Lavrentyev and B.V. Shabat. The Problem of Hydrodynamics and their Mathematical Models. Isd. Naula, Moscow (1973).

4. O.M. Byelotserkovsky and Yu.M. Davydov. The Method of Large Particles in Gas Dynamics. Izd.Nauka, Moscow (1982).

5. R.A. Jentry, R.E. Martin and B.J. Daly. "An Eulerian Differencing Method for Unsteady Compressible Flow Problems". J. Comput. Phys, 1 (1), pp. 87-118 (1966). 